\title{
Pronóstico de mortalidad y evolución en trauma pancreatoduodenal en pacientes con herida por proyectil de arma de fuego
}

\author{
Prognosis of mortality and evolution in pancreatoduodenal trauma in patients \\ with wound by projectile of firearm
}

\author{
Daniel R. Cantú-Alejoํ, Arturo Cantú-Kawas' ${ }^{1}$ Asdrúbal Guevara-Charles ${ }^{1,2}$, \\ Marco A. Hernández-Guedea ${ }^{1,2}$, Gerardo E. Muñoz-Maldonado ${ }^{1}$, Edelmiro Pérez-Rodríguez ${ }^{1}$ \\ y Francisco Reyna-Sepúlveda ${ }^{1 *}$ \\ 'Servicio de Cirugía General; 2Servicio de Urgencias. Hospital Universitario "Dr. José Eleuterio González", Universidad Autónoma de Nuevo León, \\ Monterrey, Nuevo León, México
}

\begin{abstract}
Resumen
Antecedentes: Las heridas por proyectil de arma de fuego constituyen un problema de salud pública en el mundo. Como ejemplo mencionamos que este tipo de lesiones son la primera causa de muerte en el grupo de edad de 1 a 19 años en los EE.UU., al igual que en México. Objetivo: Análisis de los factores pronósticos de mortalidad y evaluación de la evolución en pacientes con TPD por HPPAF abdominal. Método: Estudio retrospectivo, observacional y descriptivo, realizado con 49 expedientes clínicos de pacientes que fueron admitidos en el departamento de cirugía del Hospital Universitario José Eleuterio González entre los años 2011 y 2015, cuyo diagnostico fue herida por proyectil de arma de fuego con trauma pancreático (TP), duodenal (TD) o pancreatoduodenal (TPD). Resultados: Durante un periodo de recolección de 5 años se obtuvieron 49 expedientes clínicos aplicables al estudio según los criterios de inclusión, de los cuales 36 pacientes (73\%) sufrieron únicamente TD, 37 (75\%) TP y 24 (49\%) TPD. Se obtuvieron diferencias significativas para la mortalidad asociada a TD y TPD, pero no para TP. El órgano más afectado como lesión asociada a un TPD fue el hígado, seguido de las estructuras torácicas y el estómago. El factor de riesgo para mortalidad más significativo fue una estancia prolongada en la unidad de cuidados intensivos. Conclusiones: Los datos conseguidos concuerdan con los consultados, otorgando nueva estadística reproducible para futuros estudios respecto a la violencia creciente en nuestro país y alrededor del mundo.
\end{abstract}

Palabras Clave: Heridas por proyectil de arma de fuego. Mortalidad. Trauma pancreatoduodenal.

\begin{abstract}
Background: The wounds caused by the firearm projectile are published to date in a public health problem in the world. As an example, we mentioned the injuries caused by firearms are the first cause of death in the age group between 1 to 19 years in the United States, as in Mexico. Objective: Analysis of the prognostic factors of mortality and evaluation of the evolution in patients with TPD due to abdominal HPPAF. Method: Retrospective, observational, descriptive study. Helped by 49 clinical files of patients who were admitted to the department of surgery of the University Hospital José Eleuterio González, between 2011 to 2015 and
\end{abstract}

\section{Correspondencia:}

*Francisco Reyna-Sepúlveda

Avenida Francisco y Madero y Gonzalitos, s/n

Col. Mitras Centro

Fecha de recepción: 04-01-2019

C.P. 64640 , Monterrey, N.L., México

E-mail: francisco.reynas@uanl.com.mx 0009-7411/@ 2019 Academia Mexicana de Cirugía. Publicado por Permanyer. Éste es un artículo open access bajo la licencia CC BY-NC-ND (http://creativecommons.org/licenses/by-nc-nd/4.0/).

Cir Cir. 2020;88(1):82-87

Contents available at PubMed www.cirugiaycirujanos.com 
whose diagnosis was due to pancreatic trauma (TP), duodenal trauma (TD) or pancreatoduodenal trauma (TPD) by wounds caused by the firearm projectile. Results: During a collection period of 5 years, a total of 49 clinical records applicable to the study were obtained according to the inclusion criteria, of which 36 (73\%) suffered only from TD, 37 (75\%) from TP and 24 (49\%) about TPD. Significant differences were obtained for mortality associated with TD and TPD, but not for TP. The most affected organ as a lesion associated with a TPD was the liver, followed by thoracic structures and the stomach. The most significant risk factor for mortality was a prolonged stay in the intensive care unit. Conclusions: Data obtained are consistent with those consulted, providing new reproducible statistics for future studies regarding the increasing violence in our country and around the world.

Key Words: Firearm projectile injuries. Mortality. Pancreatoduodenal trauma.

\section{Introducción}

Las heridas por proyectil de arma de fuego constituyen a la fecha un problema de salud pública en el mundo. En los EE.UU. cada año fallecen por esta causa alrededor de 50,000 personas y son la primera causa de muerte en el grupo de edad de 1 a 19 años. En México se ha llegado a los 20,000 fallecimientos, aunque aquí aún se desconoce de manera amplia la epidemiologia de este tipo de lesiones ${ }^{1,2}$.

Los especialistas en este campo atribuyen el incremento en su incidencia a diferentes factores, como la violencia doméstica, el desempleo, las bajas percepciones económicas, la integración a corta edad a pandillas, el uso de drogas y la distribución no controlada de armas de fuego ${ }^{3}$.

En el Hospital Universitario José Eleuterio González, ubicado en Monterrey, en el Estado de Nuevo León, México, se reportó en 2016 la asistencia de 309 pacientes por heridas por arma de fuego originarios del mismo municipio, representando el $51 \%$ de los casos recibidos entre 2005 y 2015 . Este municipio, en el año 2011 se ubicó entre las ciudades más peligrosas del mundo, lo cual, aunque ha ido disminuyendo con el tiempo, requiere un profundo estudio al respecto ${ }^{1,4}$.

En las series de trauma abdominal, la prevalencia de trauma pancreático (TP) ha sido del 3 al 12\%, con dos tercios de las lesiones resultado de trauma penetrante y un tercio de trauma cerrado 5 . La incidencia este tipo de lesiones, aunque rara, se encuentra aumentando gradualmente con el paso de los años; se atribuye al uso de armas de fuego y accidentes en su mayoría ${ }^{6-10}$. De igual manera, el trauma duodenal (TD) se considera raro, con un compromiso menor del $5 \%$ de todos los traumas abdominales, y predominan los causadas por algún arma penetrante ${ }^{11-13}$. El trauma pancreatoduodenal (TPD) es poco frecuente; sin embargo, el requerir una pancreaduodenectomía como procedimiento quirúrgico no lo es, aunque no se ha analizado profundamente el uso de esta técnica para heridas por arma de fuego. Debido a que estas lesiones graves son poco frecuentes, hay pocas series de informes de experiencias exclusivas con este procedimiento para trauma, y ninguno ha sido publicado desde $1984^{13,14}$.

El objetivo del presente estudio es realizar un análisis de los factores pronósticos de mortalidad, evaluar la evolución en pacientes con TPD por proyectil de arma de fuego en el abdomen y realizar una comparación con el número de TD y TP respecto al de TPD, además de hacer la correlación con la mortalidad de la cantidad de órganos lesionados.

\section{Método}

El estudio es retrospectivo, observacional y descriptivo. Fue realizado a partir de 49 expedientes clínicos de pacientes, los cuales fueron admitidos en el departamento de cirugía del Hospital Universitario José Eleuterio González entre los años 2011 y 2015, presentando como principal complicación de heridas por arma de fuego intraabdominales TD, TP o TPD. Las lesiones pancreáticas y duodenales se clasificaron de acuerdo con el Comité de Escalado de Lesiones Orgánicas, parte de la American Association for the Surgery of Trauma (AAST) ${ }^{15}$.

El presente estudio fue llevado a cabo bajo la autorización del comité de investigación del Hospital Universitario José Eleuterio González. La información obtenida se recolectó dentro de una base de datos para su posterior uso en el análisis estadístico. La información privada de los pacientes se revisó solo por los autores y coautores, y como protocolo de privacidad se eliminarán los archivos digitales en un periodo de 5 años.

Los criterios de inclusión fueron:

- Operaciones realizadas dentro del Hospital Universitario José Eleuterio González.

- Pacientes con TP, TD o TPD por proyectil de arma de fuego 
Los criterios de exclusión fueron:

- Expedientes médicos sin la información concerniente para este estudio.

- Expedientes clínicos con irregularidades en su contenido o en su realización.

Se consideraron como variables el número de órganos lesionados, el grado de choque, el sexo, la edad, el número de días en internamiento, el número de días en terapia intensiva, la existencia de fracturas asociadas, la temperatura corporal, la saturación de oxígeno, la frecuencia cardiaca, la presión arterial, la frecuencia respiratoria, la puntuación ISS (Injury Severity Score), la irritación peritoneal, las lesiones en tórax, tráquea, diafragma, esófago, duodeno, páncreas, intestino delgado, intestino grueso, hígado y riñón, y sus respectivos manejos terapéuticos, tales como reparaciones primarias, resección intestinal, hemostasia e injertos vasculares, además de exclusión pilórica, las lesiones asociadas, el número de paquetes globulares usados, el internamiento en la unidad de cuidados intensivos, la estancia prolongada y el seguimiento.

Algunas de las variables analizadas no se revisaron a fondo dada su falta de significancia estadística.

Los datos se analizaron usando el programa informático Numbers ${ }^{\circledR}$ v3.6.1 (Apple Inc.). El análisis estadístico se realizó con el programa IBM SPSS Statistics v20.0 (SPSS, Inc., Armonk, NY, EE. UU.). EI análisis de riesgo se realizó tomando en cuenta el intervalo de confianza del $95 \%$.

\section{Resultados}

Durante un periodo de recolección de expedientes de 5 años se obtuvieron 49 expedientes clínicos aplicables al estudio según los criterios de inclusión, de los cuales 36 pacientes (73\%) sufrieron únicamente TD, 37 (75\%) TP y 24 (49\%) TPD.

Los valores demográficos y clínicos se muestran en la tabla 1, incluyendo lesiones orgánicas asociadas, que constituyeron el $71 \%$ de la muestra y en las que el hígado y el estómago fueron los órganos lesionados más comúnmente ligados a TPD, además de que el $92 \%$ de los TP, TD y TPD tuvieron más de dos lesiones orgánicas asociadas.

Se mostró un predominio del sexo masculino para heridas por arma de fuego. En su mayoría presentaron un daño orgánico en más de dos órganos a la vez por esta causa, pero el grado de choque solo fue $>1$ en un $26 \%$ de la muestra.

Los factores que, gracias al análisis estadístico, pudimos definir como «de riesgo» o «protectores»,

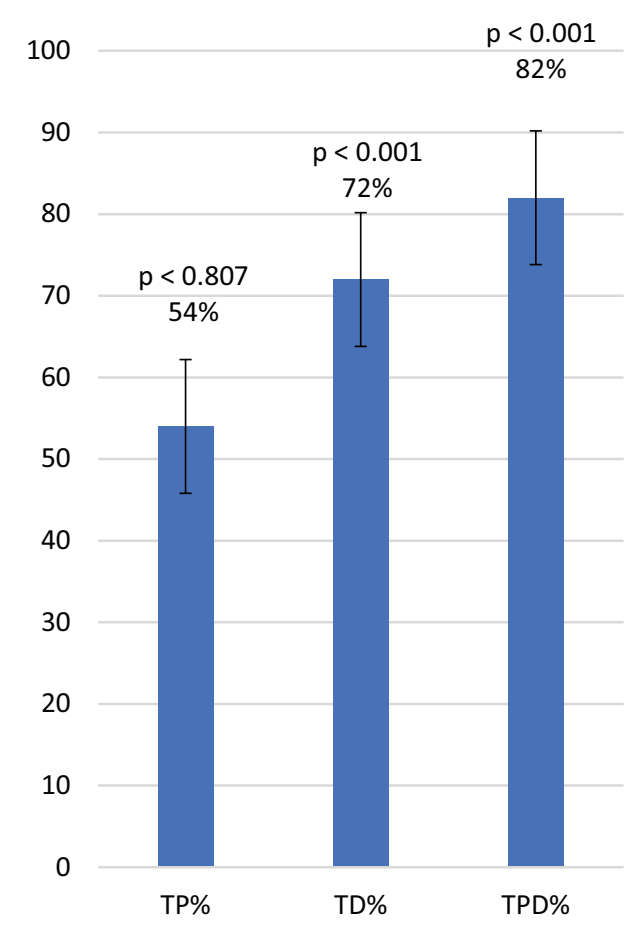

Figura 1. Comparación porcentual entre el número de traumas pancreáticos, duodenales y pancreatoduodenales. TD: traumas duodenales; TPD: traumas pancreatoduodenales; TP: traumas pancreáticos.

que obtuvieron niveles significativos (salvo en la categoría de signos vitales), se muestran en la tabla 2, El factor de riesgo para mortalidad más significativo fue una estancia prolongada en la unidad de cuidados intensivos. Además, se analizaron los signos vitales obtenidos al entrar a la sala de urgencias e intervenciones terapéuticas.

Se obtuvieron diferencias significativas para la mortalidad asociada a TD y TPD, pero no se encontraron para TP (Fig. 1).

\section{Discusión}

En la presente investigación, la prevalencia de lesiones abdominales asociadas a TP, TD o TPD ronda entre el $0.8 \%$ y un máximo del $8 \%$. Hay una clara relación anatómica entre el páncreas y el duodeno, que predispone a lesión orgánica múltiple implicando a estos dos órganos, y en la mayoría de las ocasiones a más órganos intraabdominales ${ }^{16-20}$.

Tomando en cuenta lo anterior, respecto a la mortalidad asociada a este raro evento, resultó en un porcentaje que varía entre los estudios del 18 al 50\% $\%^{17,21,22}$. En nuestro caso alcanzó una mortalidad del $53 \%$ de la muestra, lo cual concordó con la evidencia consultada 
y con estudios anteriores. Desgraciadamente, sufrimos limitaciones a lo largo del estudio en cuanto a la obtención fiable de los grados de las lesiones orgánicas.

Como era de esperar, los resultados mostraron una mayor mortalidad en los pacientes con TPD ${ }^{23}$, principalmente a causa de hemorragia interna, lesión vascular e infecciones ${ }^{7,24,25}$, en comparación con la mortalidad en los pacientes con TPD, junto con TD/TP, TD obtuvo una diferencia en la mortalidad total de la muestra; por el otro lado, TP obtuvo resultados no significativos. A pesar de este resultado, es de vital importancia remarcar la variabilidad respecto a la mortalidad entre TD y TP, ya que resultaron semejantes en la mayoría de los estudios existentes. Esto último no ha sido estudiado tan a fondo como su mortalidad amerita, ya que estas dos lesiones están tan relacionadas anatómicamente que se tratan de manera independiente de si hablamos de una lesión orgánica única o compartida ${ }^{23}$.

Dentro de las lesiones asociadas no hubo gran variedad en el estudio respecto a lo que aclaró la literatura, ocupando el primer lugar la lesión orgánica asociada estructuras vasculares, seguida de las lesiones de hígado, tórax, colon, etc. ${ }^{13,16,26}$. Sin embargo, no coincidimos en la prevalencia usualmente descrita de esta lesión respecto a las lesiones en el yeyuno y el íleon, lo cual resultó estar poco descrito en la actualidad. A pesar de esto, coincidimos en que el porcentaje de lesiones asociadas en general, dentro de nuestra muestra, fue equivalente al aproximadamente $95 \%$ descrito en la actualidad ${ }^{27}$. El método que se utilizó en gran medida para verificar el involucro orgánico fue la laparotomía exploratoria, tal como está estandarizado actualmente ${ }^{8}$.

Cavazos, et al. ${ }^{1}$ obtuvieron la prevalencia de heridas por arma de fuego en su estudio, que mostró como principal región lesionada el abdomen, aproximadamente en un $40 \%$. La presencia de temperatura mayor de $37.2{ }^{\circ} \mathrm{C}$ aumentó la mortalidad 1.3 veces en relación con la sepsis que pudo ocasionar dicha herida, ya descrita anteriormente como una de las principales complicaciones. Los demás signos vitales no demostraron aumentar la mortalidad en pacientes con TPD por herida por arma de fuego; esto toma importancia cuando consultamos la sintomatología más común al padecer un estado de choque, que es esperable en pacientes con heridas graves por arma de fuego ${ }^{28}$.

El indicador ISS de gravedad de la lesión fue sometido a investigación en el trauma abdominal por herida por arma de fuego, demostrando ser útil en la valoración de las complicaciones y de la mortalidad, y de ahí que se haya utilizado en esta investigación, al requerir una fuente fidedigna para la estandarización del estado de cada sujeto. Sin embargo, en este estudio sus resultados no fueron significativos, correlacionando este con la mortalidad. El $27 \%$ de los pacientes tenían un grado 2 de choque o superior. En la literatura, el estado de choque demostró ser un factor importante de mortalidad ${ }^{19,29,30}$. Sin embargo, en el presente estudio, el grado o la presencia de choque no mostró aumentar la mortalidad.

Se ha mencionado de manera amplia la letalidad que significa un TPD por el daño que supone, además del daño acompañado que significa el sufrir de estas lesiones intrabdominales. Por esta razón es de vital importancia la inclusión de criterios de evaluación para el ingreso de este tipo de pacientes en la unidad de cuidados intensivos ${ }^{31-34}$.

Se tomó en cuenta la estancia hospitalaria prolongada, en un hospital de tercer nivel, como aquella que cumple una duración superior a 9 días. Casi la mitad de los pacientes, el $45 \%$ (22), cumplieron el criterio anterior. Aumentó su mortalidad a 10.6 (razón de momios: 10.6) veces comparado con los que estuvieron menos días internados. Krige, et al. ${ }^{35}$, en su estudio con 75 pacientes, observaron un aumento de la mortalidad en más de la mitad de los heridos que ingresaron a la unidad de cuidados intensivos. El $63 \%$ tuvo necesidad de ingresar, lo que demostró un aumento de 8.5 veces en su mortalidad comparados con los que no se ingresaron. El $59 \%$ de los pacientes precisaron prolongar más de 5 días el ingreso en la unidad de cuidados intensivos, y esto elevó 12 veces la mortalidad. El hecho de que un paciente con TPD necesite entrar a la unidad de cuidados intensivos eleva su mortalidad, y si su ingreso se prolonga más de 5 días aumenta considerablemente, dando como resultado una mortalidad 12 veces mayor por una estancia prolongada. Esta representó la variable más importante relacionada con la mortalidad, seguida de una mortalidad 10 veces mayor por una estancia prolongada de 10 días de duración en la sala de cirugía y una mortalidad 8.5 veces mayor por una estancia superior a 5 días en la unidad de cuidados intensivos. Estos resultados pudiesen parecer obvios, pero el nivel de efecto dependiendo de la presencia o no de dichas variables, y su significancia estadística, son imprescindibles al momento de elegir métodos terapéuticos, además de prevenir complicaciones y definir de manera temprana el pronóstico de los pacientes con traumas profundos intraabdominales, sin mencionar la delicadeza que 
debe reflejarse al ingresar a estos pacientes en la unidad de cuidados intensivos.

La mortalidad por TD, TP y TPD fue comparada y los resultados obtenidos resultaron conflictivos, dada la posición anatómica del duodeno y del páncreas y su cercana relación en cuanto a lesiones. La significancia encontrada para la mortalidad respecto a TPD y TD, pero no para TP, es algo que no se replica comúnmente en la literatura, pero comparte cifras similares $(6-33 \%)^{36,37}$. Los resultados obtenidos hicieron posible establecer un pronóstico temprano para TPD respecto a TD y parcialmente para TP. Sin embargo, hacen falta más análisis para determinar el porqué de esta diferencia estadística alrededor de TP, con un mayor tamaño de muestra para la comparación de técnicas quirúrgicas y su índice de supervivencia, y además incluir las lesiones asociadas más frecuentes, por separado, de TD y TP, para definir la posible comorbilidad causada por estas, junto con sus respectivas frecuencias.

Hablando sobre técnicas quirúrgicas para la reparación de diversas heridas, demostramos un efecto benéfico para la supervivencia de procedimientos tales como la reparación gástrica, esplenectomía y duodenal, esta última con un efecto protector significativo contra la mortalidad por TD. Por otro lado, procedimientos como la exclusión pilórica y la hemostasia no demostraron modificar la mortalidad. El resto de las variables analizadas no lograron obtener significancia estadística, incluyendo procedimientos contra TP, los cuales no lograron modificar la mortalidad, salvo la realización de hemostasia pancreática al controlar el sangrado y la formación de fistulas pancreáticas, todo causado por TP, que prácticamente no modificó el pronóstico. Targarona, et al. ${ }^{38}$ mencionan que, en su estudio, la causa más frecuente de muerte fue la hemorragia intraabdominal causada por una fístula pancreática que erosionó un vaso sanguíneo o por una mala «hemostasia», que fue la causa del $37 \%$ de las muertes en los pacientes a quienes se realizó una pancreatoduodenectomía, por lo cual este último resultado retoma importancia.

\section{Conclusión}

Los datos recabados en este estudio concordaron con los proporcionados por la literatura citada, otorgando un nada despreciable nivel de significancia a los resultados, logrando el objetivo principal de llevar a cabo la interpretación de estos dentro de nuestra institución responsable. En cuanto al TPD, es considerado un evento raro cuya morbilidad se ha demostrado por la literatura en general, y ahora, de igual manera, por nuestro estudio.

\section{Conflicto de intereses}

Los autores declaran que no existe conflicto de intereses.

\section{Financiamiento}

No se recibió ningún apoyo para la realización de este trabajo.

\section{Responsabilidades éticas}

Protección de personas y animales. Los autores declaran que para esta investigación no se han realizado experimentos en seres humanos ni en animales.

Confidencialidad de los datos. Los autores declaran que han seguido los protocolos de su centro de trabajo sobre la publicación de datos de pacientes.

Derecho a la privacidad y consentimiento informado. Los autores han obtenido el consentimiento informado de los pacientes y/o sujetos referidos en el artículo. Este documento obra en poder del autor de correspondencia.

\section{Bibliografía}

1. Cavazos JC, Palacios-zertuche J, Reyna-sepúlveda F, Álvarez-Villalobos $\mathrm{N}$, Alatorre-lópez L, Muñoz-Maldonado G. Epidemiology of gunshot wounds in the University Hospital "Dr. José Eleuterio González" of the Autonomous University of Nuevo León. Cir Cir. 2017;85:41-8.

2. Cristiani Díaz G, Beltrán R. Lesiones causadas por proyectil de arma de fuego. Estudio epidemiológico en el Hospital Sharp de Mazatlán, Sinaloa. Acta Ortopédica Mexicana. 2004;18:37-40.

3. García-Valadez LR, Hernández-Téllez IE, Castellanos-Velazco CA, Ibáñez-Guerrero O, Palmieri-Bouchan RB. Epidemiología de las heridas por proyectil de arma de fuego en el Hospital Central Militar de México. Rev Sanid Milit Mex. 2015;69:204-17.

4. Martínez-Bustamante D, Pérez-Cárdenas S, Ortiz-Nieto JM, Toledo-Toledo R, Martínez-Ponce de León AR. Heridas craneales por proyectil de arma de fuego en población civil: análisis de la experiencia de un centro en Monterrey, México. Cir Cir. 2015;83:94-9.

5. Amirata E, Livingston $\mathrm{DH}$, Elcavage J. Octreotide acetate decreases pancreatic complications after pancreatic trauma. Am J Surg. 1994;168:345-7.

6. Sheng JY, Movva S. Systemic therapy for advanced soft tissue sarcoma. Surg Clin North Am. 2016;96:1141-56.

7. Debi U, Kaur R, Prasad KK, Sinha SK, Sinha A, Singh K. Pancreatic trauma: a concise review. World J Gastroenterol. 2013;19:9003-11.

8. Čečka F, Asqar A, Jon B, Kočí J, Šubrt Z, Ferko A. Gun-shot injuries to the abdomen involving the pancreas. Acta Chir Orthop Traumatol Cech. 2012;79(5):455-8.

9. Biffl WL, Moore EE, Croce M, Davis JW, Coimbra R, Karmy-Jones R, et al. Western trauma association critical decisions in Trauma: Management of pancreatic injuries. J Trauma Acute Care Surg. 2013;75:941-6.

10. Carrazana AAJ, Alonso JRC, Quiala LNM, Mendoza AA. Resultados del tratamiento quirúrgico en un traumatismo pancreaticoduodenal. Rev Cuba Cir. 2011:50:490-9.

11. Rickard MJFX, Brohi K, Bautz PC. Pancreatic and duodenal injuries: keep it simple. ANZ J Surg. 2005;75:581-6.

12. Seamon MJ, Pieri PG, Fisher CA, Gaughan J, Santora TA, Pathak AS, et al. A ten-year retrospective review: does pyloric exclusion improve clinical outcome after penetrating duodenal and combined pancreaticoduodenal injuries. J Trauma. 2007;62:829-33. 
13. Asensio JA, Petrone P, Roldán G, Pak-art R, Salim A. Pancreatic and duodenal injuries. Complex and lethal. Scand J Surg. 2002;91:81-6.

14. Peraza Acosta M. Pancreatoduodenectomia o procedimiento de Whipple. Rev Medica Costa Rica y Centroam. 2014;71:559-62.

15. Linsenmaier U, Wirth SR, Reiser M, Körner M. Diagnosis and Classification of Pancreatic and Duodenal Injuries in Emergency Radiology. RadioGraphics. 2008:28(6):1591-602.

16. Feliciano DV, Burch JONM, Spjut-patrinely V, Mattox KL, Jordan GL. Abdominal gunshot wounds an urban trauma center's experience with 300 consecutive patients. Ann Surg. 1987;208:362-7.

17. Antonacci N, Di Saverio S, Ciaroni V, Biscardi A, Giugni A, Cancellieri F, et al. Prognosis and treatment of pancreaticoduodenal traumatic injuries: which factors are predictors of outcome? J Hepatobiliary Pancreat Sci. 2011;18:195-201.

18. Petrone P, Moral Álvarez S, González Pérez M, Ceballos Esparragón J, Marini CP. Orientación terapéutica del traumatismo pancreático: revisión de la literatura. Cir Esp. 2016;95:123-30.

19. DiGiacomo L, Schreiber MA. Penetrating pancreatic injury. Curr Trauma Rep. 2015;1:85-91.

20. Dorado Ferrufino CH, López Terán A, Camacho Apaza J. Características del manejo del trauma duodenal en pacientes atendidos en el Complejo Hospitalario Viedma. Gac Med Boliv. 2011;34:25.

21. Martin TD, Feliciano DV, Mattox KL, Jordan GL Jr. Severe duodenal injuries. Treatment with pyloric exclusion and gastrojejunostomy. Arch Surg. 1983;118:631-5.

22. Sharma AK. Management of pancreaticoduodenal injuries. Indian J Surg. 2012;74:35-9

23. O'Reilly DA, Bouamra O, Kausar A, Malde DJ, Dickson EJ, Lecky F. The epidemiology of and outcome from pancreatoduodenal trauma in the UK, 1989-2013. Ann R Coll Surg Engl. 2015;97:125-30.

24. Bokhari F, Phelan H, Holevar M, Brautigam R, Collier B, Como JJ, et al. EAST Guidelines for the diagnosis and management of pancreatic trauma. Eastern Association for the Surgery of Trauma. Practice Management Guidelines Committee; 2009. Disponible en: https://www.east.org/ Content/documents/practicemanagementguidelines/pancreatic-trauma-diagnosis-and-management.pdf

25. Ghosh SK. One stage emergency pancreatoduodenectomy for isolated injury to pancreatic head following blunt abdominal trauma: case report and review of literature. Bull Emerg Trauma. 2013;1:127-9.
26. Lahiri R, Bhattacharya S. Pancreatic trauma. Ann R Coll Surg Engl. 2013;95:241-5.

27. Vasquez JC, Coimbra R, Hoyt DB, Fortlage D. Management of penetrating pancreatic trauma: an 11-year experience of a level-1 trauma center. Injury. 2001;32:753-9.

28. Ramos Xavier L, Alves Crespo M, Soares de Oliveira CM, De Freitas Baldez $L$. Tratamiento inicial de heridas por proyectil de arma de fuego. A propósito de un caso clínico. Rev Esp Cirug Oral y Maxilofac. 2008;30:115-20.

29. Shah AA, Rehman A, Shah SJ, Haider AH, Zogg CK, Zafar SN, et al. Abdominal gunshot wounds - a comparative assessment of severity measures. J Surg Res. 2015;198:334-9.

30. Krige JE, Kotze UK, Setshedi M, Nicol AJ, Navsaria PH. Prognostic factors, morbidity and mortality in pancreatic trauma: a critical appraisal of 432 consecutive patients treated at a level 1 trauma centre. Injury. 2015;46:830-6.

31. Magaña Sánchez IJ, Belmonte Montes C, Noyola Villalobos H, Cabello Pasini R. Traumatismo pancreato-duodenal combinado. Cirujano General. 2000;M(70):226-31.

32. Moore EE, Cogbill TH, Malangoni MA, Jurkovich GJ, Champion HR, Gennarelli TA, et al. Organ injury scaling, II: pancreas, duodenum, small bowel, colon, and rectum. J Trauma - Inj Infect Crit Care. 1990;30:1427-9.

33. Asensio JA, Petrone P, Rolda G, Kuncir E. Pancreaticoduodenectomy: a rare procedure for the management of complex pancreaticoduodenal injuries. J Am Coll Surg. 2003;197:937-42.

34. Asensio JA, Petrone P, Kimbrell B, Kuncir E. Trauma duodenal. Técnica y manejo. Rev Colomb Cir. 2006;21:4-14.

35. Krige JE, Kotze UK, Setshedi M, Nicol AJ, Navsaria PH. Surgical management and outcomes of combined pancreaticoduodenal injuries: analysis of 75 consecutive cases. J Am Coll Surg. 2016;222:737-49.

36. Pandey S, Niranjan A, Mishra S, Agrawal T, Singhal BM, Prakash A, et al. Retrospective Analysis of Duodenal Injuries: A Comprehensive Overview. Saudi J Gastroenterol. 2011;17:142-4.

37. Dave S, London S. Pancreatic trauma. StatPearls Publishing; 2018. Disponible en: https://www.ncbi.nlm.nih.gov/books/NBK459365/.

38. Targarona J, Pando E, Garatea R, Vavoulis A, Montoya E. Morbilidad y mortalidad postoperatorias de acuerdo al "factor cirujano" tras duodenopancreatectomía. Cir Esp. 2007;82:219-23. 